\title{
Sciences Basic to Psychiatry: AUTP Guidelines
}

The Standing Committee of the Association of University Teachers of Psychiatry (AUTP) have recently prepared guidelines for teachers and students on sciences basic to psychiatry. This followed a request from the College and these guidelines are now being published in the Bulletin. They have been considered by the Education Committee and by Council who commend them to members of the College.

The guidelines do not refer to the important subjects of dynamic or descriptive psychopathology, not because the AUTP wished to exclude them from basic sciences, but because they did not present the same problems to teachers or students. It is hoped, however, that the Standing Committee will consider these subjects later. The Chairman of the AUTP, in referring these guidelines to the College, has pointed out that at present basic sciences are taught in the first year of training. There are some disadvantages with this arrangement, as it might encourage trainees to think of the subject as something to be learned and forgotten, and it makes it unnecessarily difficult to integrate basic sciences and clinical teaching. In some ways it might be more satisfactory to teach basic sciences over the whole of the three years of training and examine them in the Membership papers. In drawing up these guidelines, however, the AUTP considered what would be appropriate in the present circumstances of an examination after one year.

The future of the MRCPsych Examination is currently under review, and the Court of Electors has set up a special working party with its membership drawn from the Court and the Examinations Sub-Committee who will consider and review possible future changes in the Examination, including the Preliminary Test. If major changes are introduced into the Examination, advance notice will be given of this, so that trainees will not be disadvantaged by being inappropriately prepared, and trainers will have had sufficient time to reorganize their courses and training programmes.

In the meantime the College would wish to commend, particularly to tutors, the helpful advice in these guidelines, emphasizing that they are not a formal College syllabus but a guide to some of the core elements of the sciences basic to psychiatry.

Thomas H. BewLeY

Dean

\section{Guidelines for Students and Teachers}

Psychiatrists need to learn about basic sciences if they are to have a full understanding of psychiatric disorders and their treatment, and they need to be able to assess and assimilate advances in knowledge which occur during their professional careers. Any syllabus in basic sciences for psychiatrists training in this country assumes a basic medical education of the kind provided in the United Kingdom, including an elementary knowledge of behavioural sciences and statistics. A trainee whose medical education was in another country may not have studied a comparable range of subjects, and it may be necessary to arrange some additional instruction to bring him or her to this starting point.

Because knowledge is advancing rapidly in all the sciences basic to psychiatry, any syllabus will require regular revision. For the same reason it is essential that the trainee should learn to evaluate evidence of new discoveries and not merely be informed about the present state of knowledge. To achieve this, the student should study a few subjects in detail at the same time as he acquires a reasonably broad knowledge of relevant parts of the field. A useful teaching method is to consider critically scientific papers in the subject.

It is not the purpose of this document to provide a justification for basic science teaching to trainees in psychiatry. We assume that there is general agreement that some scientific knowledge is essential. Nor have we attempted a detailed justification for the choice of each of the disciplines included here: they too appear to be rather generally agreed. Instead the purpose of these guidelines is to indicate the balance between disciplines and the selection of topics within each discipline. The consensus views were arrived at by a working party of the AUTP (Drs R. Palmer, M. Weller and G. Hay). The working party obtained evidence from 52 correspondents, who included both psychiatrists and teachers of the relevant basic sciences. The curriculum that follows is not intended to restrict the trainee's interests; he should certainly have covered the subjects in this list, but he should be encouraged to pursue additional topics which interest him.

Many subjects can be considered basic to psychiatry, but the following have a serious claim for inclusion and are part of most existing courses of instruction: neuroanatomy, neuropathology, neurochemistry, neurophysiology (including neuroendocrinology), psychology, ethology, social anthropology, sociology, epidemiology, statistics and research methods. These guidelines do not cover phenomenological and dynamic psychopathology, which form part of the preliminary test but present fewer problems to clinicians, in the definition of the syllabus. With such a long list there is an obvious risk that teaching will be superficial and fragmented. For this reason, teaching should emphasize the inter-relations of different disciplines, and the examiners should ask some questions which relate to this. At the same time, within each subject some topics should be selected which can illustrate the scientific method and the ways of 
thought which are characteristic of that discipline. The aim is to achieve the middle ground between undue narrowness and unrealistic breadth.

Teaching should ideally be shared between informed clinicians (psychiatrists and clinical psychologists) and those working in the basic sciences. Videotape material could be incorporated usefully to illustrate experimental methods.

\section{Paychology and Ethology"}

Although psychology and psychiatry are closely related, the trainee cannot cover every aspect of the subject and should choose a few to study in more detail than the rest so that he understands the methods of thought and enquiry in the subject. Teaching should not try to hide controversy. Psychology would be expected to occupy about a third of the basic science course.

A knowledge of human psychological development is required, with an emphasis on child development and ageing. Special attention should be given to the effects of separation from parents in human children and in animals. The development of sexual behaviour and gender role should be understood. In teaching these subjects, attention should also be paid to the psychodynamic aspects of development and mental functioning.

The psychology of learning is a subject that is suitable for study in detail to illustrate the experimental methods available in psychology. The minimum requirement includes a basic knowledge of classical and operant conditioning in animals and man, latent learning, extinction and stimulus and response generalization. The trainee should know of attempts to understand neuroses and other behaviour disorders as the result of abnormal learning and be able to evaluate these critically. Insightful learning and social learning, including modelling, should also be covered. The psychology of educational learning and teaching should be understood in so far as they relate to the imparting of information and the teaching of new skills to patients and their relatives.

The study of memory provides another useful illustration of different experimental approaches to a single problem, e.g., psychological studies of normal human memory, ablation studies in animals, acetyl choline abnormalities in dementia and loss of function in Korsakoffs psychosis. Cerebral localization and lateralization should also be covered.

Motivation and arousal and their influence on performance should be understood. The psychology of emotion should be known well (it overlaps with teaching in physiology). Psychophysiological studies should include a critical evaluation of work on biofeedback.

"Psychology as applied to psychiatry is the subject of a joint working party of the British Psychological Society and the Royal College of Psychiatrists, and our recommendations may need to be revised in the light of their report, though we would maintain the need to be selective.
Cognitive and perceptual psychology need not be studied in detail; a basic understanding will include concepts of perceptual constancy, sensory threshold, attention, set, vigilance, reaction time and information processing. The psychology of human skills need not be known in detail.

Basic concepts and methods of psychometrics must be understood, viz., reliability, validity, sensitivity and methods of scaling and test construction. The limitations of the different kinds of test must be understood, especially with reference to testing intelligence and personality, and some knowledge is required of approaches to individual differences other than personality testing. A basic knowledge is also required of methods for rating behaviour and interview responses.

Although social psychology need not be covered comprehensively, the concept of attitude should be understood, together with factors which alter attitudes, non-verbal communication, social skills and group behaviour. (This subject overlaps aspects of sociology.)

Only basic concepts and methods of investigation in ethology need be known, principally the concepts of imprinting, bonding, fixed action patterns and releasers.

\section{Neurosciences}

There is now a substantial body of established knowledge in these disciplines, so that it is necessary to be selective despite their evident importance. The trainee must learn a critical approach that will equip him to assess further advances in these rapidly changing subjects. Together, these subjects should occupy about $\mathbf{4 0}$ per cent of the course. This figure assumes a knowledge of basic concepts equivalent to that covered in an average pre-clinical course, and this may require some preliminary revision.

In neuroanatomy the gross anatomy of the brain and spinal cord and peripheral nervous system should be revised as a basis for neurological diagnosis. In addition, the anatomy of structures associated with emotion and the higher functions of the brain should be known thoroughly. A basic knowledge is required of the various cell types and cellular organelles.

The study of neuropathology overlaps that of neuroanatomy. As well as the topics listed above, an elementary knowledge is required of the use of electron microscopy, tissue culture and enzyme histochemistry as investigative techniques. The principal neuropathological changes in degenerative disorders, cerebrovascular diseases and other conditions leading to dementia should be known.

In neurophysiology, knowledge of neurons, synapses and receptors should be revised, but need not go beyond the level of an average pre-clinical course. The physiology of the motor and sensory systems and the autonomic nervous system should be understood to a similar level. The physiology of emotion should be known thoroughly. Cerebral localization and the effects of injury should also be understood (this topic overlaps with neuroanatomy and 
psychology). The anatomy and physiology of the neuroendocrine system should be revised from a psychiatric perspective, and knowledge is required of the physiology of sleep and of pain. Aspects of memory, learning and emotion which appear here illustrate the way in which physiological and psychological studies complement one another. A basic knowledge of the physiological basis of the EEG is required, together with the physiology of sleep.

A basic knowledge is required of the neurochemistry of aminoacids, proteins, nucleic acid, enzymes and enzyme regulation, but this need not go beyond the level of an average pre-clinical course. The biochemistry of neurotransmitters and hallucinogens, which overlaps teaching in pharmacology, should be known thoroughly.

In pharmacology, basic principles of pharmacokinetics and drug action should be known thoroughly in relation to the main groups of drugs used in psychiatry (antidepressants, major tranquillizers, sedative/hypnotics, amphetamines, lithium and anticonvulsants). The mode of action and effects of these drugs must be known thoroughly (though details of the uses are not required for the preliminary test). The pharmacology of placebo response and the methods of drug trials overlap with the syllabus on psychology and on research methods.

The pharmacology of neurotransmitters and small peptides should be known, and one or more (e.g., dopamine) studied in enough detail to illustrate the way in which neurochemistry, neurophysiology, pharmacology and behavioural studies can contribute to the solution of a single problem and allow the student to appreciate the thinking that lies behind the investigations and the uncertainties that remain.

\section{Sociology and Social Anthropology}

Although these topics have obvious relevance to psychiatry, the body of well established scientific evidence is so far not sufficiently substantial to justify a major part of the course. About 10 per cent is appropriate. In sociology topics of relevance include social class, roles (including sick role), stigma, illness behaviour, the family and institutions. The student should know a few examples of social factors in disease, though details are not required, and be able to discuss the relation of psychiatry and medicine to society. As in other branches of science, one or two subjects should be taught in sufficient depth to illustrate the ways of thought in the discipline and show points of disagreement. Methods of quantifying life events should be understood. Knowledge of social anthropology need not be extensive but should include an understanding of cultural differences in family and social roles, particularly as they apply to ethnic minorities in this country; and the range of human development as it is shown by its variations in different societies.

\section{Genetics}

Candidates should have the level of knowledge required in an average pre-clinical course about genes, chromosomes, cell division and principles of inheritance and be able to illustrate the concepts with examples relevant to psychiatry. The student should be able to give examples of the main modes of iuheritance and chromosomal abnormality, but detailed knowledge of the disorders will not be expected at this stage. The principles of twin and adoption studies must be known, with appropriate clinical examples. The subject will occupy about 5 per cent of the course in addition to any time needed to revise the basic knowledge referred to above.

\section{Epidemiology, Statistics and Research Methods}

For the preliminary test the trainee should concentrate on the principles of epidemiology; details of the findings of psychiatric epidemiology will be learnt later. Therefore, the time devoted to epidemiology need not be substantial, say 5 per cent of the total. The traince should understand the concepts of incidence, prevalence and population at risk, and the problems associated with classification (including the ICD), the definition of cases and the production of morbidity statistics. He should understand how epidemiology can be used to measure prevalence and determine aetiology. Easily available measures of morbidity should be understood, together with their limitations, and knowledge of case register methods and screening techniques is required.

The learning of statistics and research methods should occupy about 5 to 10 per cent of the total time. The trainee should understand the purpose and limitations of the common methods of analysis and be able to recognize when they are being misused. It is not necessary to know the details of the computations used in these tests (except for the chi square). The trainee should be able to define the basic concepts of descriptive statistics, such as mean, mode, range, normal distribution and standard deviation, and understand the concepts of sampling, probability, null hypothesis and statistical significance. He must be able to distinguish between ordinal and interval data and understand the uses of non-parametric statistics; perform and interpret the chi square test and understand the uses of correlation, regression and analysis of variance and covariance. He should appreciate the relation between statistical methods and research design. He should have a general understanding of research methods as they apply in psychiatric investigations, including trials of treatment. 